\title{
A study to find the characteristic imaging findings of various ring- enhancing lesions using MR imaging
}

\author{
Ramachandra V ${ }^{\mathbf{1}}$, Neil Mekala I. ${ }^{2 *}$, Chandra Mataparthy A. ${ }^{3}$, Chandra T. ${ }^{4}$ \\ DOI: https://doi.org/10.17511/ijmrr.2020.i02.04 \\ ${ }^{1}$ Ramachandra V, Assistant Professor, Department of Radiodiagnosis, GSL Medical College, Rajahmundry, Andhra Pradesh, India. \\ 2* Indra Neil Mekala, Assistant Professor, Department of Radiodiagnosis, GSL Medical College, Rajahmundry, Andhra Pradesh, India. \\ 3 Anish Chandra Mataparthy, Resident, Department of Radiodiagnosis, GSL Medical College, Rajahmundry, Andhra Pradesh, India. \\ 4 T Jaya Chandra, Scientist Incharge, Central Research laboratory, GSL Medical College, Rajahmundry, Andhra Pradesh, India.
}

Introduction: Multiple ring-enhancing lesions are one of the most commonly encountered neuroimaging abnormalities. A study was conducted to find the characteristic imaging findings of various ring-enhancing lesions on MRI. Materials and Methods: It was a prospective study conducted in the department of radiodiagnosis, GSL Medical College. All age groups of both gender with cerebral ring-enhancing lesions detected in contrast MR studies were taken up for spectroscopy. Patients with a history of claustrophobia and individuals with metallic implants insertion, cardiac pacemakers, and metallic foreign body in situ were excluded. The MRI scan was performed MRI PHILIPS ACHIEVA HDxt $1.5 \mathrm{~T}$, the active shielded superconducting magnet of 1.5 telsa magnetic field using SENSE coils for the acquisition of images. Results: Out of 30 study participants, under $<45$ years category tuberculoma was the common (37\%) lesion whereas in $>45$ years category metastases was common (15\%), 44\% were female and 56\% were males. Depicts T1 signal intensity showed hypointense signal intensity on T1W sequence in 65 (95.5\%) cases. Only $4.6 \%$ of cases showed isointense signal intensity on T1W image. T2 signal intensity in $73.5 \%$ of cases showed hyperintense signal intensity. Conclusions: MRI is an excellent, non-ionizing imaging modality with multiplanar imaging capabilities for excellent grey-white matter differentiation.

Keywords: Lesion, Participant, Gender, Age

Corresponding Author

Indra Neil Mekala, Assistant Professor, Department of Radiodiagnosis, GSL Medical College, Rajahmundry, Andhra Pradesh, India.

Email: gslcentralresearchlab@gmail.com
How to Cite this Article To Browse
Ramachandra V, Mekala IN, Mataparthy AC, Chandra TJ. A study to find the characteristic imaging findings of various ring-enhancing lesions using MR imaging. Int J Med Res Rev. 2020;8(2):159-163.

Available From

https://ijmrr.medresearch.in/index.php/ijmrr/article/ view/1171

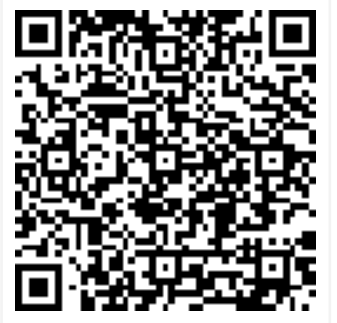

\section{Introduction}

Manuscript Received 2020-02-25

Conflict of Interest No
Multiple ring-enhancing lesions are one of the most commonly encountered neuroimaging abnormalities. Widely available imaging

Review Round 1
2020-03-04
Funding
Nil

Review Round 1 $\mathrm{Nil}$
Review Round 2 2020-04-08

Ethical Approval YES
Techniques, computed tomography (CT) and magnetic resonance imaging (MRI) are used to detect these lesions. A wide range of etiologies may present as cerebral multiple ring-enhancing lesions $[1,2]$. On neuroimaging, these lesions appear as

Review Round 3

Accepted 2020-04-13

(C) 2020 by Ramachandra V Indra Neil Mekala, Anish Chandra Mataparthy, T Jaya Chandra and Published by Siddharth Health Research and Social Welfare Society. This is an Open Access article licensed under a Creative Commons Attribution 4.0 International License https://creativecommons.org/licenses/by/4.0/ unported [CC BY 4.0]. 
Hypodense or isodense mass lesions on noncontrast $\mathrm{CT}$ studies. After contrast administration, there is a ring or a homogeneous disk-like enhancement within the region of hypodensity. The enhancing lesions are often of variable sizes and are usually surrounded by varying amounts of vasogenic edema. Typically, the ring-enhancing lesions are located at the junction of the gray and white matter, but they could be located in the sub-cortical area, deep in the brain parenchyma or may even be superficial [3]. Magnetic resonance spectroscopy (MRS) provides information about the possible extent and nature of changes on a routine MRI scan by analyzing the presence and / or ratio of tissue metabolites such as NAA, creatine, choline, and lactate etc. Widespread usage of faster MRS applications with a higher signal-to-noise ratio (SNR) and spatial resolution, allows us to detect functional metabolic changes, which provides more data to understand the exact nature of the tumor and the morphological and physiological changes occurring in surrounding brain parenchyma. Longitudinal studies have demonstrated that HMRS is useful in monitoring disease progression and treatment effects. MR spectroscopy also has a prognostic implication [4]. With these as the study was conducted with an aim to find the characteristic imaging findings of various ring-enhancing lesions on MRI.

\section{Materials and Methods}

Setting: The study was conducted in the department of radiodiagnosis, GSL Medical College, Rajahmundry.

Study period and study design: Study was conducted from January 2014 to December 2014. It was a Hospital-based It was a prospective study.

Study method: Random sampling was considered in this study.

Inclusion criteria: All the age groups of both gender with cerebral ring-enhancing lesions detected in contrast MR studies were taken up for spectroscopy. All patients with incidentally diagnosed ring-enhancing lesions by CT.

Exclusion criteria: Patients with a history of claustrophobia and individuals with metallic implants insertion, cardiac pacemakers, and metallic foreign body in situ were excluded from the study.

Study population: Patients of both gender of all age groups who were referred to the department of
Radiodiagnosis with clinically suspected and identified on neuroimaging of cerebral ringenhancing lesions.

Ethical issue: The study protocol was approved by the institutional ethics committee.

All those patients who were included in the study were provided with a consent form in the local language and patients were explained about the diagnostic modalities. Written consent was obtained from those patients who voluntarily willing to participate in the study. The MRI scan was performed MRI PHILIPS ACHIEVA HDxt $1.5 \mathrm{~T}$ possesses an Ultra-compact, Superconducting, Active shielded superconducting magnet of 1.5 telsa magnetic field using SENSE coils for the acquisition of images. Conventional spin-echo sequences, axial T1, and T2 weighted. Coronal T2; SagittalT1; Postcontrast axial, coronal and sagittal; DWI; T2 GRE single and multi-voxel spectroscopy was performed at TEof144. The voxel is placed on the lesions that cover the maximum area of the lesion in both single and multivoxel. PRESS and T1post contrast sequence as localization sequence with $5 \mathrm{~mm}$ thickness. Other sequences were used as and when required.

Statistical analysis: All the statistical analysis is done using SPSS software version 21 and MS excel 2007. Descriptive statistics in the form of mean, percentage were used.

\section{Results}

During the study period, a total of 30 participants were included in the study. Age-wise the participants were divided in to <45years and $>45$ years categories which were $71 \%$ (48) and $29 \%$ (20) respectively. Under $<45$ years category, abscess was diagnosed in $13 \%$ (9) members, gliomas in 3\% (2) cases, neurocysticercosis in $18 \%$ (12) cases, tuberculoma in $37 \%$ (25). Whereas in $>45$ years category, Glioblastoma multiforme was diagnosed in 3\% (2) cases, glioma in $1.5 \%$ (1), metastases in $15 \%$ (10) cases, neurocysticercosis in $3 \%$ (2) cases and tuberculoma in $7.4 \%$ (5) cases. Totally, abscess was diagnosed in $13 \%$ (9) cases, glioblastoma multiforme in 3\% (2), gliomas in $4.5 \%$ (3), metastases in 15\% (10), neurocysticercosis in $20 \%$ (10) and tuberculoma was diagnosed in $44 \%$ (30) cases (Table 1). Gender wise 44\% (30) were female participants and 56\% (38) were male and the male-female ratio was 1.27 . Among females, an abscess was diagnosed in $9 \%$ cases, glioblastoma 
Multiforme, gliomas in $1.5 \%$ (1) each receptively, metastases in $4.4 \%$ cases, neurocysticercosis in $13 \%$ and tuberculoma in $15 \%$ (10) cases. Among the male, an abscess was diagnosed in $4.4 \%$ cases, glioblastoma multiforme in $1.5 \%$ (1), gliomas in $3 \%$, metastases in $10 \%$ cases, neurocysticercosis in $7 \%$ and tuberculoma in 30\% (20) cases (Table 2). Depicts $\mathrm{T} 1$ signal intensity showed hypointense signal intensity on T1W sequence in $65(95.5 \%)$ cases. Only $3(4.6 \%)$ out of $68(100 \%)$ cases showed isointense signal intensity on T1W image. T2 signal intensity out of which $50(73.5 \%)$ cases showed hyperintense signal intensity on T2W images and $17(25 \%)$ cases showed hypointense signal intensity, all the 17 cases were tuberculomas. Only $1(1.5 \%)$ case showed heterogeneous signal intensity on $\mathrm{T} 2 \mathrm{~W}$ sequence.

Table-1: Age-wise incidence of various pathological lesions present as ring-enhancing lesions among the study participants; $\mathbf{n}(\%)$.

\begin{tabular}{|l|l|l|l|}
\hline \multirow{2}{*}{ Lesion } & \multicolumn{2}{c|}{ Age } & \multirow{2}{*}{ Total } \\
\cline { 2 - 3 } & $<45$ years & $>45$ years & \\
\hline Abscess & $9(13)$ & 0 & $9(13)$ \\
\hline Glioblastoma multiforme & 0 & $2(3)$ & $2(3)$ \\
\hline Gliomas & $2(3)$ & $1(1.5)$ & $3(4.5)$ \\
\hline Metastases & 0 & $10(15)$ & $10(15)$ \\
\hline Neurocysticercosis & $12(18)$ & $2(3)$ & $14(20)$ \\
\hline Tuberculoma & $25(37)$ & $5(7.4)$ & $30(44)$ \\
\hline Total & $48(71)$ & $20(29)$ & $68(100)$ \\
\hline
\end{tabular}

Table-2: Gender wise distribution of various pathological lesions among the study participants; $\mathbf{n}(\%)$

\begin{tabular}{|l|l|l|l|}
\hline \multicolumn{1}{|c|}{ Lesion } & \multicolumn{1}{|c|}{ Female } & \multicolumn{1}{c|}{ Male } & \multicolumn{1}{c|}{ Total } \\
\hline Abscess & $6(9)$ & $3(4.4)$ & $9(13)$ \\
\hline Glioblastoma multiforme & $1(1.5)$ & $1(1.5)$ & $2(3)$ \\
\hline Gliomas & $1(1.5)$ & $2(3)$ & $3(4.4)$ \\
\hline Metastases & $3(4.4)$ & $7(10)$ & $10(15)$ \\
\hline Neurocysticercosis & $9(13)$ & $5(7)$ & $14(21)$ \\
\hline Tuberculoma & $10(15)$ & $20(30)$ & $30(44)$ \\
\hline Total & $30(44)$ & $38(56)$ & $68(100)$ \\
\hline
\end{tabular}

\section{Discussion}

Magnetic resonance imaging (MRI) is a noninvasive, multiplanar, and highly accurate method with better inherent contrast that demonstrates the lesion accurately. MRI provides an accurate assessment of the brain changes in various ringenhancing lesions, for accurate diagnosis and introduction of immediate treatment. Out of the 68 patients who were evaluated, tuberculomas 30 $(44.1 \%)$ is the most common pathology followed by
NCC $14(20.5 \%)$, abscesses $9(13.2 \%)$, metastasis $10(14.7 \%)$ and primary brain tumors 5 (7.3\%).In a study conducted by Schwartz et al., $40 \%$ of cases were gliomas. The higher incidence of tuberculomas is probably due to the higher prevalence of tuberculosis in India [5]. Sixty-eight patients were evaluated, whose age group was divided into below 45 years and above 45 years. The highest incidence of ring-enhancing lesions (RELs) was found in below 45 years age group accounting for $70.5 \%$ of cases and less commonly in the above 45 age group constituting $29.5 \%$. Sixty-eight patients were evaluated of which $38(55.9 \%)$ were males and 30 $(44.1 \%)$ were females. Out of sixty-eight cases, tuberculomas were seen in $30(44 \%)$ patients. In this, 25 cases were in $<45 \mathrm{yrs}$ age group and 20 were men. Single lesions were seen in 13 cases $(43 \%)$ and both conglomerate and multiple lesions were seen in $57 \%$. They were seen as conglomerate lesions which are hypointense on both $\mathrm{T} 1$ and $\mathrm{T} 2$ weighted images. These lesions show iso to hyperintense ring on 12 cases. Diffusion-weighted imaging showed partial or complete restriction seen in $77 \%$. Tae Kyung Kim et al. stated in intracranial tuberculoma, granulomas showed slightly hyperintense rim on T1 weighted images. Granulomas showed slightly heterogenous iso or hypointensity with small markedly hypointense foci [6]. Gupta RK et al. Mentioned that there was not much difference in ADC values between tuberculomas and tubercular abscesses [7]. The present study was compared with Jayasundar et al., which concluded that the presence of lipid can be used for differentiating tuberculomas from both non-specific IG and NCC [8]. Whereas Vasudev MK et al., concluded that intracranial tuberculomas are characterized by relatively short $\mathrm{T} 2$ relaxation times compared to normal gray matter, decreased MTR, and no restriction of diffusion [9]. Neurocysticercosis was seen in $20.5 \%$ cases, most of them presented with seizures, and very few presented with other complaints like headache, vomiting, and ataxia, etc. All the cases were $<45$ years, 9 were female. The present study findings were compared with Amaral $L$ et al. which concluded that MR imaging is sensitive and specific in the analysis of different forms of NCC and differentiating other etiologies [10]. Whereas Chang $\mathrm{KH}$ et al., stated MRI is superior to CT in the evaluation of CNS parasitic infections [11]. HR Martinez R RangelGuerra et al., mentioned that MR is sensitive in diagnosing active NCC [12]. Haris M, Gupta RK et al. stated that post-contrast perfusion indices such 
Ask (trans) and $v$ (e) helps in differentiating infective from neoplastic brain lesions [13]. The current study doesn't have any pregnant women as compared to Savita R Singhal et al., reported NCC presenting in the second trimester of pregnancy with convulsions [14]. Kumar et al. stated the Cho/Cr ratio was less than 1.1 in all NCC and more than 1.2 in all tuberculoma which was similar to the present study findings [15]. Halmes et al. described the appearance of abscesses on MR. The current study correlated our findings with those described and distinguishes the edema, central necrosis, and the characteristic pattern of peripheral enhancement of the abscess capsule [16]. The current study findings correlated with Zee CS et al., which studied and concluded MRI-DWI helps in early diagnosis of cerebral abscesses and evaluation of treatment outcome [17]. The current study findings were similar to Tsui EY et al., which concluded that conventional MRI along with DWI and MRS establishes diagnosis [18]. Mishra AM et al., which stated restricted diffusion on DWI with reduced ADC is highly suggestive of brain abscess [19]. Out of sixty-eight cases, two were GBM. Both patients were $<45 y r s$, presented with seizures, headache, and vomiting. Single lesions were identified in both cases. Midline shift was seen in both cases. All the cases were hypointense on $\mathrm{T} 1$ and hyperintense. Diffusion restriction present in both cases. On contrast administration, all the cases showed complete, thick, and irregular ring enhancement. Most of the cases showed high Choline and reduced NAA levels. The present study findings were compared and similar to Tsougos I et al., which stated that Peritumoral $\mathrm{N}$-acetyl aspartate (NAA)/creatine ( $\mathrm{Cr}$ ), choline (Cho)/Cr and Cho/NAA helps in differentiating glioblastomas from intracranial metastases [20]. Whereas Hung Wen Kao et al. mentioned that DWI may serve as an early surrogate marker of therapeutic efficacy by implying persistent cellular density in the tumors where high cellularity may impede free water diffusion, resulting in a reduction of apparent diffusion coefficient (ADC) values. Generally, lower $A D C$ values correspond to increased cellularity and high-grade gliomas. Improved analysis methods indeed enhance the role of DWI as a biomarker of tumor cellularity for the diagnosis and monitoring treatment response. Advanced MR imaging technique along with conventional MR can potentially help evaluate the underlying key histopathological features of gliomas by showing the physiologic changes and metabolic activities, thus
Improving diagnosis and tumor grading provides a better understanding of the tumor behavior and treatment response [21].

\section{Limitations}

Due to the small sample size, the current study results cannot be generalized. Hence further studies with a large sample size needed.

\section{Conclusion}

MRI is an excellent, non-ionizing imaging modality with multiplanar imaging capabilities for excellent grey-white matter differentiation and identifying the precise anatomical location and the exact extent of lesions. The data so far indicated MRI findings of selected ring-enhancing lesions were significantly correlated with the age of patients. Also, this study found that benign RELs were seen predominantly in less than 45 years of age.

\section{What does the study add to the existing knowledge}

The ring-enhancing lesions can be diagnosed by MRI, which is an excellent imaging technique.

\section{Author's contribution}

Dr. V Ramachandra: Literature survey, paper writing, data analysis Dr. Indra Neil Mekala: Complete idea, statistical analysis, paper writing Dr.

Anish Chandra Mataparthy: Benchwork, sample collection, literature search, paper writing $\mathbf{D r}$. T Jaya Chandra: Data analysis, paper writing, statistical analysis

\section{Reference}

01. Omuro AM, Leite CC, Mokhtari K, Delattre JY. Pitfalls in the diagnosis of brain tumours. Lancet Neurol. 2006;5(11)937-948.

doi: [Article] [Crossref]

02. Cunliffe $\mathrm{CH}$, Fischer I, Monoky D, Law M, Revercomb C, Elrich S, et al. Intracranial lesions mimicking neoplasms. Arch Pathol Lab Med. 2009;133(1)101-123.

doi: [Article] [Crossref]

03. Smirniotopoulos JG, Murphy FM, Rushing EJ, Rees JH, Schroeder JW. Patterns of contrast enhancement in the brain and meninges. Radiograph. 2007;27(2)525-551.

doi: [Article] [Crossref] 
04. Bulakbasi N. Clinical applications of proton MR spectroscopy in the diagnosis of brain tumours. Spectro. 2004;18(2)143-153.

doi: [Article] [Crossref]

05. Schwartz KM, Erickson BJ, Lucchinetti C. Pattern of T2 hypointensity associated with ringenhancing brain lesions can help to differentiate pathology. Neuroradiol. 2006;48(3)143-149. doi: [Article] [Crossref]

06. Kim TK, Chang KH, Kim CJ, Goo JM, Kook MC, Han $\mathrm{MH}$. Intracranial Tuberculoma- Comparison of MR with Pathologic Findings. AJNR Am J Neuroradiol. 1995;16(9)1903-1908.

[Crossref]

07. Gupta RK, Husain M, Vatsal DK, Kumar R, Chawla S, Husain N. Comparative evaluation of magnetization transfer MR imaging and in vivo proton MR spectroscopy in brain tuberculomas. Magn Reson Imaging. 2002;20(5)375-381.

doi: [Article] [Crossref]

08. Jayasundar R, Singh VP, Raghunathan P, Jain $K$, Banerji AK. Inflammatory granulomas: evaluation with proton MRS NMR. Biomed. $1999 ; 12(3) 139-144$.

doi: [Article] [Crossref]

09. Vasudev MK, Jayakumar PN, SrikanthSG, Nagarajan K, Mohanty. A Quantitative magnetic resonance technique in the evaluation of intracranial tuberculomas. Acta Radiol. 2007;48(2)200-206.

doi: [Article] [Crossref]

10. Amaral L, Maschietto M, Maschietto R, Cury $R$, Ferreira NF et al. Unusual manifestations of neuro cysticercosis in MR imaging: analysis of 172 cases. Arq Neuropsiquiatr. 2003;61(3A):533-41.

doi: [Article] [Crossref]

11. Chang $\mathrm{KH}$, Han MH. MRI of CNS parasitic diseases. J Magn Reson Imaging. 1998;8(2)297-307. doi: [Article] [Crossref]

12. HR Martinez. R Rangel Guerra, G Elizondo, J Gonzalez, LE Todd et al. MR Imaging in neurocysticercosis- a study of 56 cases. Am J Neuroradiol.

1989;10(5)1011-1019 [Crossref]
13. Haris M, Gupta RK, Singh A, Husain N, Husain M et al. Differentiation of infective from neoplastic brain lesions by dynamic contrast-enhanced MRI. Neuro Radiol. 2008;50(6)531-540. doi: [Article] [Crossref]

14. Singhal SR, Nanda S, Singhal SK. Neuro cysticercosis as an important differential of seizures in pregnancy- two case reports. J Med Case Reports. $2011 ; 5 ; 206$. doi: [Article] [Crossref]

15. Kumar A, Kaushik S, Tripathi RP, Kaur P, Khushu $S$. Role of in vivo proton MR spectroscopy in the evaluation of adult brain lesions- Our preliminary experience. Neurol India. $2003 ; 51 ; 474-478$.

[Crossref]

16. Halmes $A B$, Zimmerman RD, Morgello $S$, Weingarten $\mathrm{K}$, Becker RD et al. MR Imaging of brain abscesses. Am J Roenlogenol AJR. 1989;152(5)1073-1085. doi: [Article] [Crossref]

17. Zee CS, Segall HD, Boswell W, Ahmadi J, Nelson $M$ et al. MR imaging of neuro cysticercosis. J Comput Assist Tomogr. 1988;12;927-934. doi: [Article] [Crossref]

18. Tsui EY, Chan JH, Cheung YK, Lai KF, Fong D, Ng $\mathrm{SH}$. Evaluation of cerebral abscesses by diffusion-weighted MR imaging and MR spectroscopy. Comput Med Imaging Graph. 2002;26(5)347-351. doi: [Article] [Crossref]

19. Mishra AM, Gupta RK, Jaggi RS, Reddy JS, Jha DK et al. Role of diffusion-weighted imaging and in vivo proton magnetic resonance spectroscopy in the differential diagnosis of ring-enhancing intracranial cystic mass lesions. J Comput Assist Tomogr. 2004;28(4)540-547. doi: [Article] [Crossref]

20. Tsougos I, Svolos P, Kousi E, Fountas K, Theodorou K, Fezoulidis I, et al. Differentiation of gioblastoma multiforme from metastatic brain tumor using proton magnetic resonance spectroscopy, diffusion and perfusion metrics at 3 T. Cancer Imaging. 2012;12(3)423-436. doi: [Article] [Crossref]

21. Kao HW, Chiang SW, Chung HW, Tsai FY, Chen CY. Advanced MR Imaging of Gliomas- An Update. Biomed Res Int. 2013;2013;970586. doi: [Article] [Crossref] 